\title{
eSTIMe : outil de géovisualisation pour accompagner l'analyse des mobilités quotidiennes
}

Aline Menin, Paule-Annick Davoine, Sonia Chardonnel, Michael Ortega, Étienne Duble et Luciana Nedel

\section{OpenEdition}

\author{
Journals
}

Édition électronique

URL : https://journals.openedition.org/mappemonde/6349

DOI : 10.4000/mappemonde.6349

ISSN : $1769-7298$

Éditeur

UMR ESPACE

\section{Référence électronique}

Aline Menin, Paule-Annick Davoine, Sonia Chardonnel, Michael Ortega, Étienne Duble et Luciana Nedel, " eSTIMe : outil de géovisualisation pour accompagner l'analyse des mobilités quotidiennes »,

Mappemonde [En ligne], 131 | 2021, mis en ligne le 08 juillet 2021, consulté le 14 juillet 2021. URL : http://journals.openedition.org/mappemonde/6349; DOI : https://doi.org/10.4000/mappemonde. 6349

Ce document a été généré automatiquement le 14 juillet 2021. 


\title{
eSTIMe : outil de géovisualisation pour accompagner l'analyse des mobilités quotidiennes
}

\author{
Aline Menin, Paule-Annick Davoine, Sonia Chardonnel, Michael Ortega, \\ Étienne Duble et Luciana Nedel
}

\section{Contexte et motivation}

1 Mieux comprendre et visualiser les pratiques de mobilité quotidienne est devenu un enjeu pour définir des politiques publiques locales favorisant des mobilités durables et plurielles dans des environnements urbains. L'enjeu est d'améliorer la qualité de vie des habitants en réduisant les effets indésirables des mobilités (congestion, pollution), tout en garantissant l'accessibilité aux territoires pour ses populations (diversification des offres de transport et de services). Au-delà du besoin de connaissances sur les flux pour calibrer les infrastructures de transport, il est nécessaire de comprendre comment les programmes d'activités des individus influencent leurs besoins de ressources urbaines (services, commerces, loisirs) et comment ils impactent leur mobilité et leur fréquentation des secteurs d'un territoire métropolitain. Dans ce contexte, beaucoup d'experts ont besoin de manipuler des données de mobilité plus ou moins standardisées - sans être des spécialistes du transport - pour extraire des connaissances synthétiques et facilement exploitables. Pour cela, nous proposons eSTIMe (Spatio-Temporal Exploration of Individual Mobility data) (Menin, 2020), un outil de géovisualisation qui vise à faciliter l'exploration et l'analyse de données de mobilité afin que les utilisateurs puissent traiter dans un même environnement trois familles de questionnements sous-jacents au phénomène de la mobilité urbaine quotidienne.

- Une première question est de savoir comment les habitants d'un territoire se déplacent au quotidien et quels processus d'échanges entre les lieux cela génère. Les déplacements sont alors l'objet d'intérêt et l'on cherche à analyser leur quantité (à l'échelle de l'individu et de la population), leurs modalités (modes de transport, modes d'accompagnement, motifs) en faisant varier ces valeurs selon des catégories d'habitants ou des types d'espaces. Toujours 
partant des déplacements, on peut plus particulièrement s'interroger sur la structure spatiale que ces mouvements dessinent sur le territoire. On s'intéresse alors aux liens entre les lieux de départ et les lieux d'arrivée des déplacements en construisant des «flux » par agrégation des déplacements selon un découpage du territoire en zones d'origines et de destinations (Bahoken, 2011).

- Un autre questionnement lié à la mobilité concerne l'estimation de la variation au cours du temps de la population présente sur un territoire. Ceci permet de mieux appréhender la réalité des usages des sous-espaces d'un territoire en tenant compte des caractéristiques des populations qui les fréquentent et des types d'activités qu'ils y effectuent. Ce sont alors ces «sous-espaces» du territoire qui sont analysés et pour lesquels sont produits des indicateurs dérivés des données de mobilité pour évaluer la présence des personnes à différents moments de la journée. Doivent être spécifiés dans ce cas les délimitations des sous-espaces et le pas de temps auquel on veut observer des variations de présence. Une meilleure connaissance des rythmes quotidiens des territoires est rendue possible par ces analyses (Miranda et al., 2017).

- Enfin, une dernière question est relative aux programmes d'activités motivant la mobilité. Il s'agit de comprendre comment les individus ordonnent au fil du temps leurs activités et leurs déplacements dans le contexte spatial du territoire. L'attention est ici portée sur la séquence des activités spatialisées, aussi nommée «trajectoire quotidienne ». L'analyse de l'ensemble des trajectoires peut alors viser à dresser des typologies (Robette, 2011) qui décrivent la variété des programmes d'activités qui sous-tendent et expliquent le besoin de déplacements.

\section{Les données mobilisées}

2 L'environnement eSTIMe est conçu de manière générique pour accueillir différentes sources de données de mobilité. Pour l'heure, nous avons implémenté les données des enquêtes ménages déplacements (EMD) réalisées auprès d'un échantillon représentatif de la population de trois régions métropolitaines en France : l'édition de 2010 de Grenoble (CEREMA, 2010) où 16641 individus résidents de 354 communes ont été interrogés; l'édition de 2015 de Lyon où 28230 individus résidents de 569 communes ont été interrogés (CEREMA, 2015); l'édition 2018 de Rennes, où environ 11000 individus résidents de 392 communes ont été interrogés (CEREMA, 2017) Cette enquête permet de connaître la mobilité, restreinte aux jours ouvrables, des individus (de 5 ans et plus) qui appartiennent à un ménage résidant dans les communes de la zone d'étude. Les données décrivent les caractéristiques des ménages (localisation, logement, motorisation, etc.) et des personnes (âge, sexe, profession, etc.) ainsi que l'ensemble des déplacements réalisés la veille du jour d'enquête pour toutes les personnes du ménage.

3 L'espace du territoire des enquêtes est divisé en secteurs de tirage, où un nombre $\mathrm{x}$ (identique pour chaque secteur) de ménages a été enquêté. Des coefficients de redressement, basés sur la commune de résidence, la taille et la motorisation des ménages, sont ensuite appliqués à l'échantillon pour représenter la population mère, à savoir l'ensemble des habitants de la région enquêtée. Les secteurs de tirage correspondent à l'unité statistiquement représentative minimale pour la diffusion des résultats et leur superficie varie selon la densité de la population. Par ailleurs, un découpage de zones fines (environ 500 zones pour Grenoble et Rennes et 1000 zones 
pour Lyon) est proposé aux personnes lors de l'enquête pour décrire précisément la localisation de départ et d'arrivée de chacun de leur déplacement.

4 Pour permettre une exploration des indicateurs de mobilité à plusieurs niveaux géographiques, des découpages supplémentaires, basés sur l'agrégation des secteurs de tirages, sont proposés. Ainsi, sont disponibles un zonage d'une trentaine de secteurs et un zonage d'une dizaine de secteurs dont les délimitations recoupent en partie certains zonages administratifs à l'échelle départementale et intercommunale (voire des arrondissements intra-urbains pour Lyon).

\section{Les visualisations}

L'environnement eSTIMe intègre un ensemble de visualisations (Menin et al., 2020) qui permettent de représenter et explorer les indicateurs de mobilité dérivés des données aux différents niveaux spatiaux et temporels et en fonction des différents attributs. Elles sont définies comme suit :

- Le diagramme de flux (voir figure 1) permet de visualiser les indicateurs de flux. Nous représentons les flux entre les secteurs au moyen d'un diagramme de cordes. Les arcs représentent les secteurs géographiques qui sont reliés entre eux par des rubans dont l'épaisseur décrit le volume d'échanges de flux entre deux secteurs. Cette visualisation peut être générée de façon globale pour l'ensemble des flux, ou de façon plus spécifique pour les flux selon leurs motifs ou modes de transport. Ces derniers sont représentés par la couleur des rubans. La visualisation peut aussi être générée pour l'ensemble du territoire, pour un secteur donné, ou pour une sélection de jusqu'à 10 secteurs choisis de façon interactive sur une carte.

Figure 1. Le diagramme de flux pour le motif travail

Estimated flows exchanges within the region of Grenoble

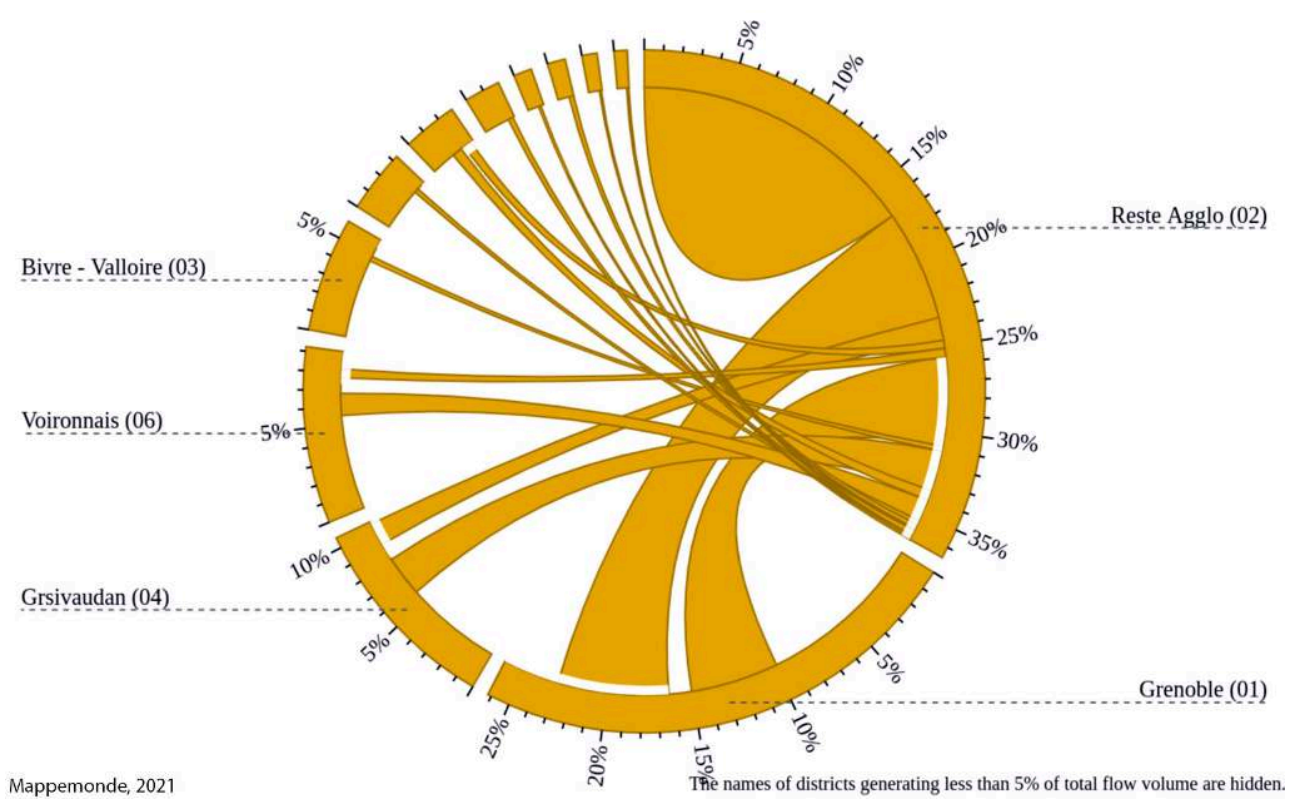

- La roue de mobilité (voir figure 2) se présente sous la forme d'un diagramme circulaire qui simule une horloge de 24 heures. Elle comporte deux anneaux formés par 24 rectangles 
représentant des périodes d'une heure. Le cercle extérieur de la roue affiche, pour chaque heure, l'indicateur du taux de mobilité, défini comme le rapport du nombre d'individus distincts en déplacement vers ou à l'intérieur du secteur. Le cercle intérieur affiche, pour chaque heure, la distribution des individus en déplacement par motif/mode de transport. Enfin, le diagramme circulaire au centre présente cette même distribution thématique sur 24 heures ou par heure.

Figure 2. La roue de la mobilité

Trip Purposes of moving people in Oisans (84) around the clock

Activity / Trip Purpose
Home
Leisure
Shopping
Education
Business
Personal busines
Escort Trips
Other purposes
Moving People (\%)
0.00 - 0.80
0.80 - 4.58
4.58 - 11.96
11.96 - 15.86
15.86 - 20.45
20.45 - 23.59
23.59 - 28.43

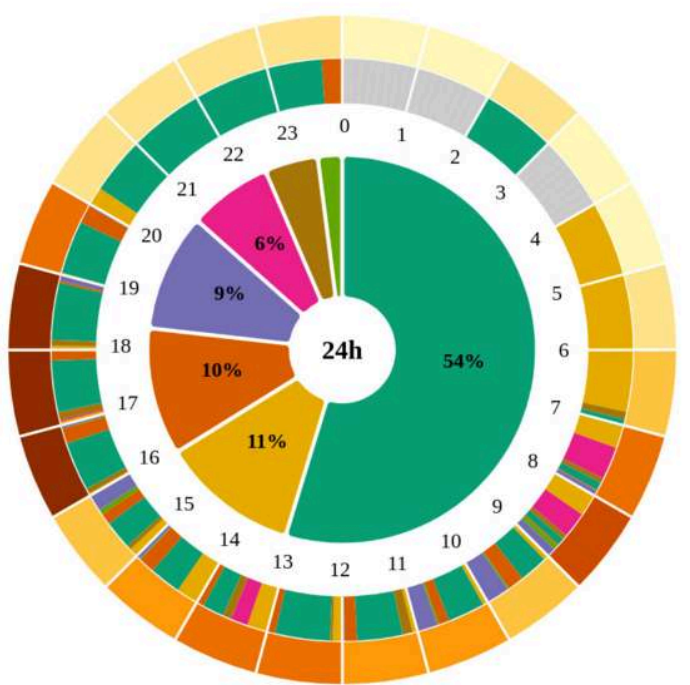

Mappemonde, 202

- Les cartes choroplèthes ou de symboles proportionnels (voir figure 3) qui s'appuient sur les règles de sémiologie cartographique classique (Bertin, 1983) servent à visualiser les indicateurs de présence des individus, densité de présence, indice d'attractivité et variation de la population des secteurs. La présence des individus par activité est définie par l'effectif des individus distincts qui ont visité ce secteur chaque heure pour effectuer chaque activité. La densité de présence est définie comme le rapport du nombre d'individus distincts ayant visité le secteur chaque heure et la surface du secteur en kilomètres carrés. La variation relative de la population permet d'identifier l'évolution de pertes et gains relatifs de population d'un secteur pour les 24 heures de la journée à travers le rapport du nombre d'individus distincts ayant visité le secteur à chaque heure et le nombre de résidents du secteur. L'attractivité est calculée sur 24 heures et elle est définie comme le rapport entre le nombre d'individus distincts ayant visité le secteur et le nombre de résidents du secteur, pondéré par la valeur globale de ce même rapport pour l'ensemble de l'enquête. Un rapport supérieur à 1 signifie que la densité moyenne de la population présente du secteur est plus importante que la densité de la population résidente, un rapport inférieur à 1 montre l'inverse (André-Poyaud et al., 2006). 
Figure 3. La carte choroplèthe

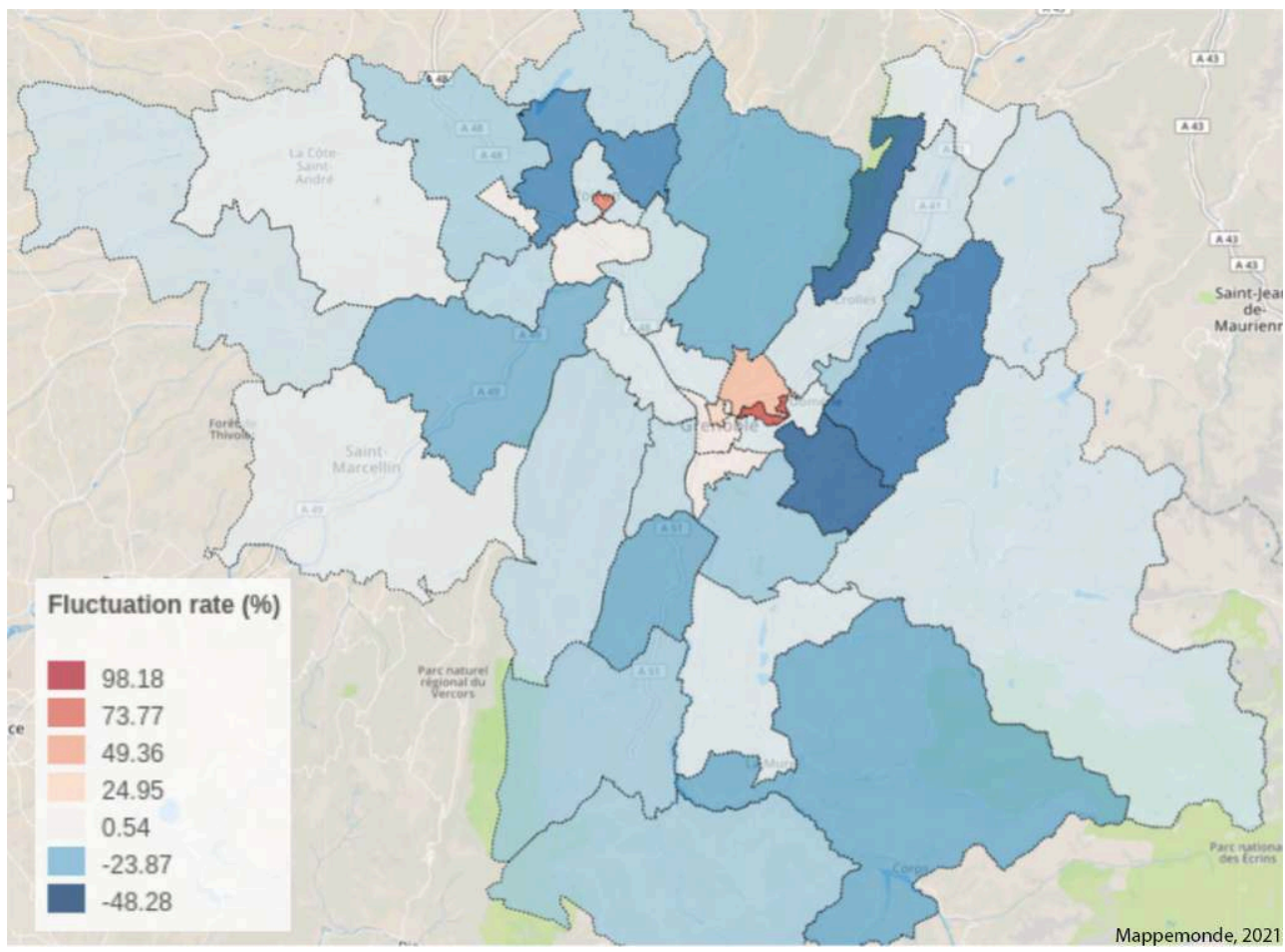

- Le chronogramme (voir figure 4) sert à visualiser les indicateurs de présence et de taux de mobilité. En effet, il représente la distribution des individus en fonction des catégories d'activités réalisées au cours de la journée. Il se présente sous la forme d'un graphique à bandes empilées, pour lequel l'axe des abscisses représente le temps et l'axe des ordonnées représente la proportion de personnes par activité (Pistre et al., 2020). Cette visualisation sert aussi à montrer la distribution des activités ou modes de transport de l'ensemble des individus appartenant aux différentes classes issues d'une typologie des programmes d'activités ou visitant un secteur donné.

Figure 4. Le chronogramme

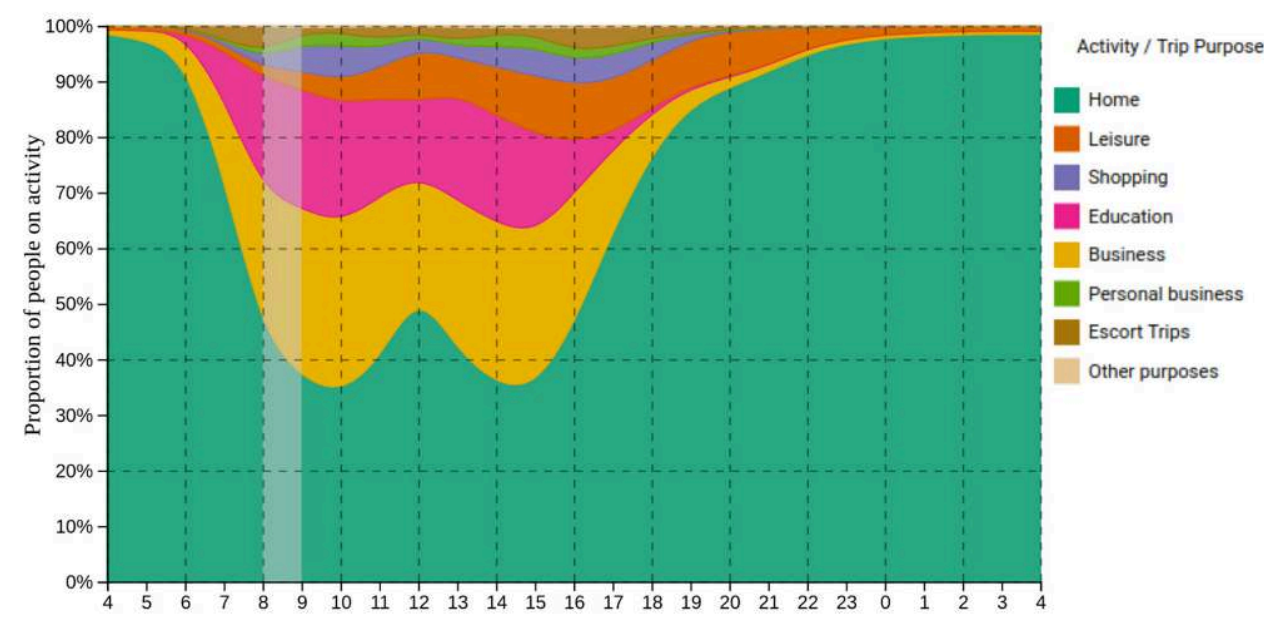

Mappemonde, 2021

- Les actogrammes (voir figure 5) montrent l'ordre temporel des activités d'une personne au cours d'une journée (Chardonnel et al., 2010). Chaque individu de l'enquête est représenté par une ligne et chaque ligne est composée de segments de couleurs différentes. La couleur 
correspond à l'activité réalisée. L'abscisse représentant le déroulement temporel des trajectoires montre le moment et la durée de chaque activité. La superposition des individus en ligne, autrement appelée tapis, permet de comparer visuellement les séquences entre elles. Cette visualisation en tapis est proposée à l'utilisateur pour représenter les séquences regroupées dans les classes d'une typologie issue d'une analyse de similarités de séquences basée sur l'usage de la Dynamic Hamming Distance (Lesnard, 2010).

Figure 5. L'actogramme

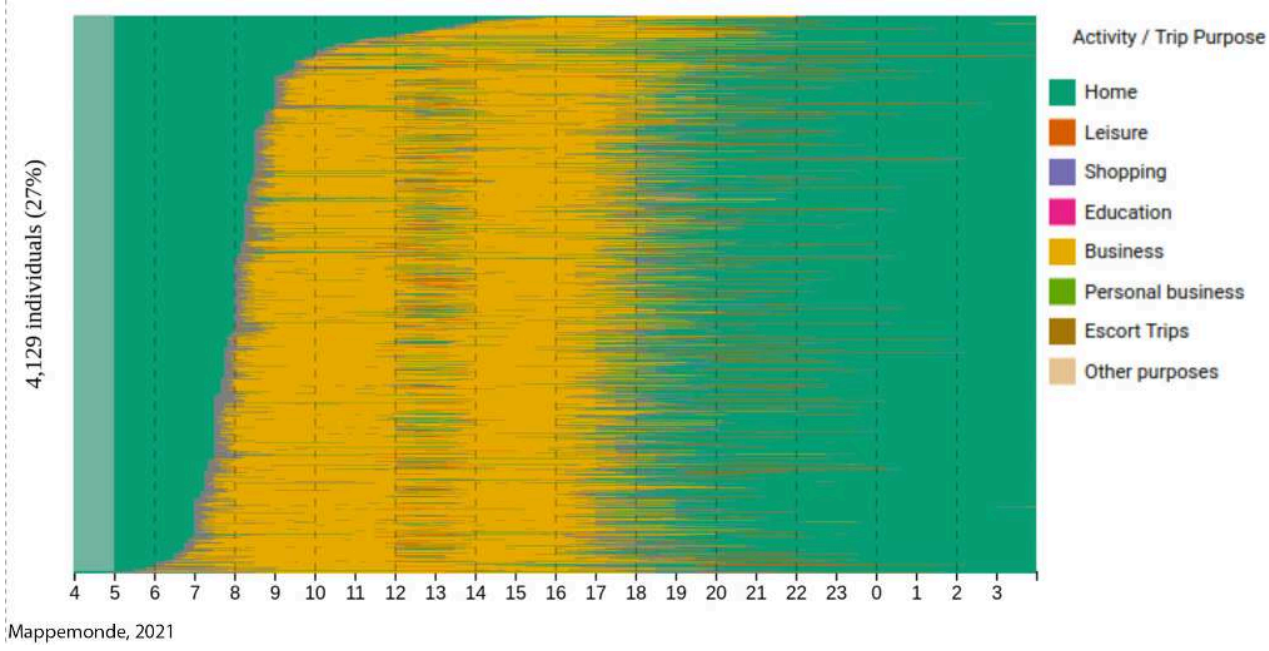

- Le cube spatio-temporel (STC) (voir figure 6) projette les trajectoires quotidiennes individuelles dans le contexte géographique du territoire étudié. Il permet d'explorer simultanément les formes et l'étendue des trajectoires individuelles dans l'espace et dans le temps. Nous intégrons dans le cube la possibilité de caractériser les trajectoires au moyen de la couleur pour visualiser les activités et/ou les modes de transport qui les composent. L'utilisateur peut explorer un ensemble de trajectoires dans le cube en les sélectionnant en fonction des caractéristiques socio-démographiques des individus. Le cube permet en outre d'explorer la dimension spatiale des typologies des programmes d'activités en affichant les trajectoires spatio-temporelles de chaque classe. 

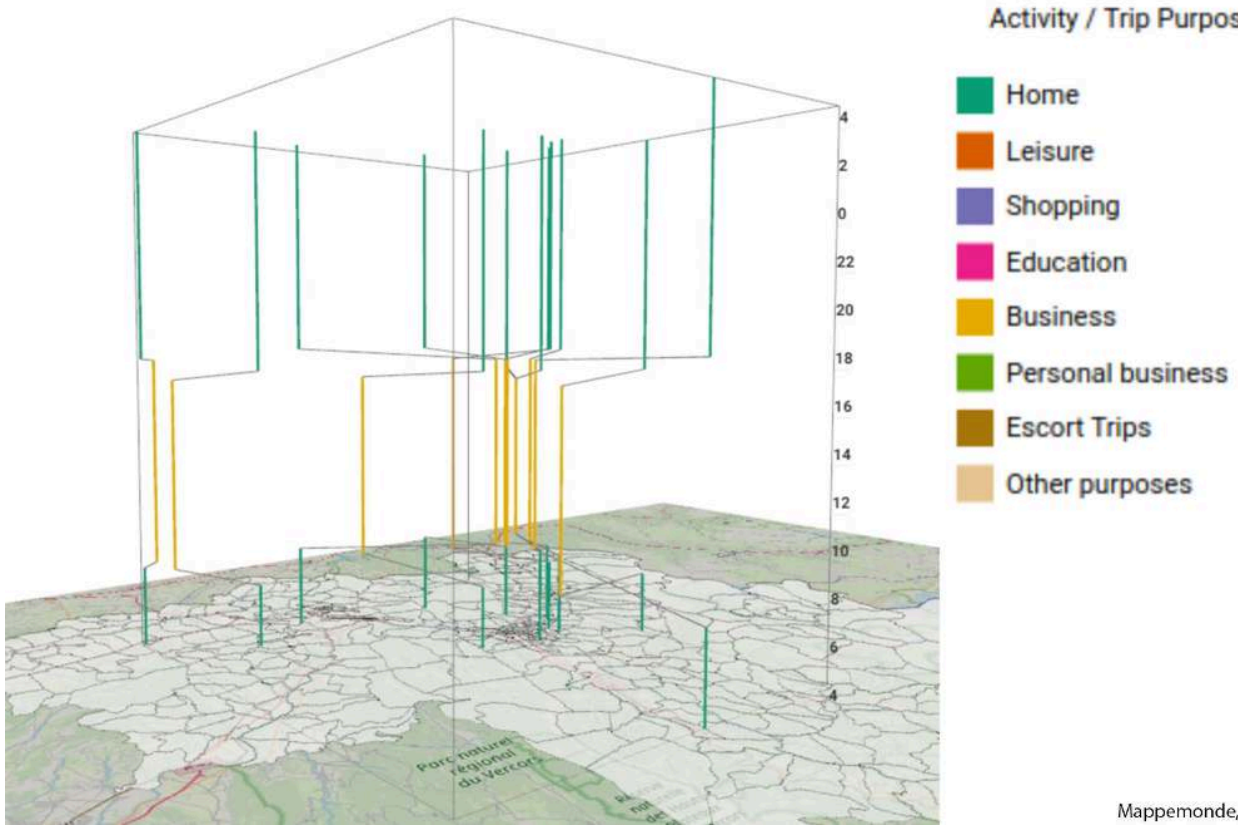

\section{L'exploration interactive}

L'environnement eSTIMe est basé sur l'utilisation d'au moins deux écrans : le premier s'affiche sur une tablette et contient l'interface interactive (appelée le Contrôleur) qui intègre des fonctionnalités d'exploration des données basée sur l'entrée tactile et tangible du dispositif. Le second écran prend la forme d'un tableau de bord, composé de quatre fenêtres, initialement vierges, sur lequel l'utilisateur affiche les indicateurs de son choix, personnalisant ainsi son espace de visualisation en fonction de ses besoins d'analyse. L'utilisateur peut avoir plusieurs tableaux de bord (et donc plusieurs écrans) connectés au contrôleur. L'interface du contrôleur est divisée en quatre espaces interactifs (voir figure 7):

- Le sélecteur de données est proposé sous deux formats :

- un menu à base d'onglets qui sert à connecter, déconnecter et contrôler les visualisations sur les tableaux de bord. Chaque onglet reproduit la disposition des fenêtres de chaque tableau de bord, ce qui permet à l'utilisateur de contrôler chaque fenêtre indépendamment via des boutons de sélection et un sous-menu de construction d'indicateurs.

- une barre de navigation qui sert à contrôler directement les indicateurs visualisés sur la carte et le cube spatio-temporel affichés sur la tablette.

- L'explorateur spatio-temporel est supporté par le dispositif mobile pour permettre à l'utilisateur de construire les indicateurs en sélectionnant les secteurs géographiques sur une carte tactile (disponible aux différents niveaux de sectorisation) et les tranches horaires sur une ligne temporelle, soit de manière tactile, soit en utilisant la technique d'interaction tangible. La sélection du temps et de l'espace est gérée de façon indépendante, afin de permettre la comparaison de plusieurs visualisations basées sur des indicateurs portant sur différents secteurs et/ou différents créneaux temporels. L'utilisateur peut aussi figer le temps sur une ou plusieurs visualisations, tout en préservant l'animation temporelle sur les restantes. 
-L'explorateur des trajectoires aussi disponible sur la tablette représente les trajectoires quotidiennes des individus sur le cube spatio-temporel. L'interaction fournie par l'entrée tactile permet la sélection des trajectoires, mettant en valeur les zones fines par lesquelles l'individu est passé au cours de la journée. Cette visualisation est synchronisée avec les actogrammes pour favoriser l'analyse complémentaire des formes et étendues des trajectoires dans l'espace-temps avec la structuration temporelle des programmes d'activités.

- L'historique qui conserve une trace de l'activité de l'utilisateur dans l'environnement, et peut être utilisé pour annuler/rétablir des actions et pour mieux comprendre le raisonnement de l'analyste en examinant son utilisation du système.

Figure 7. Les composants interactifs d'eSTIMe

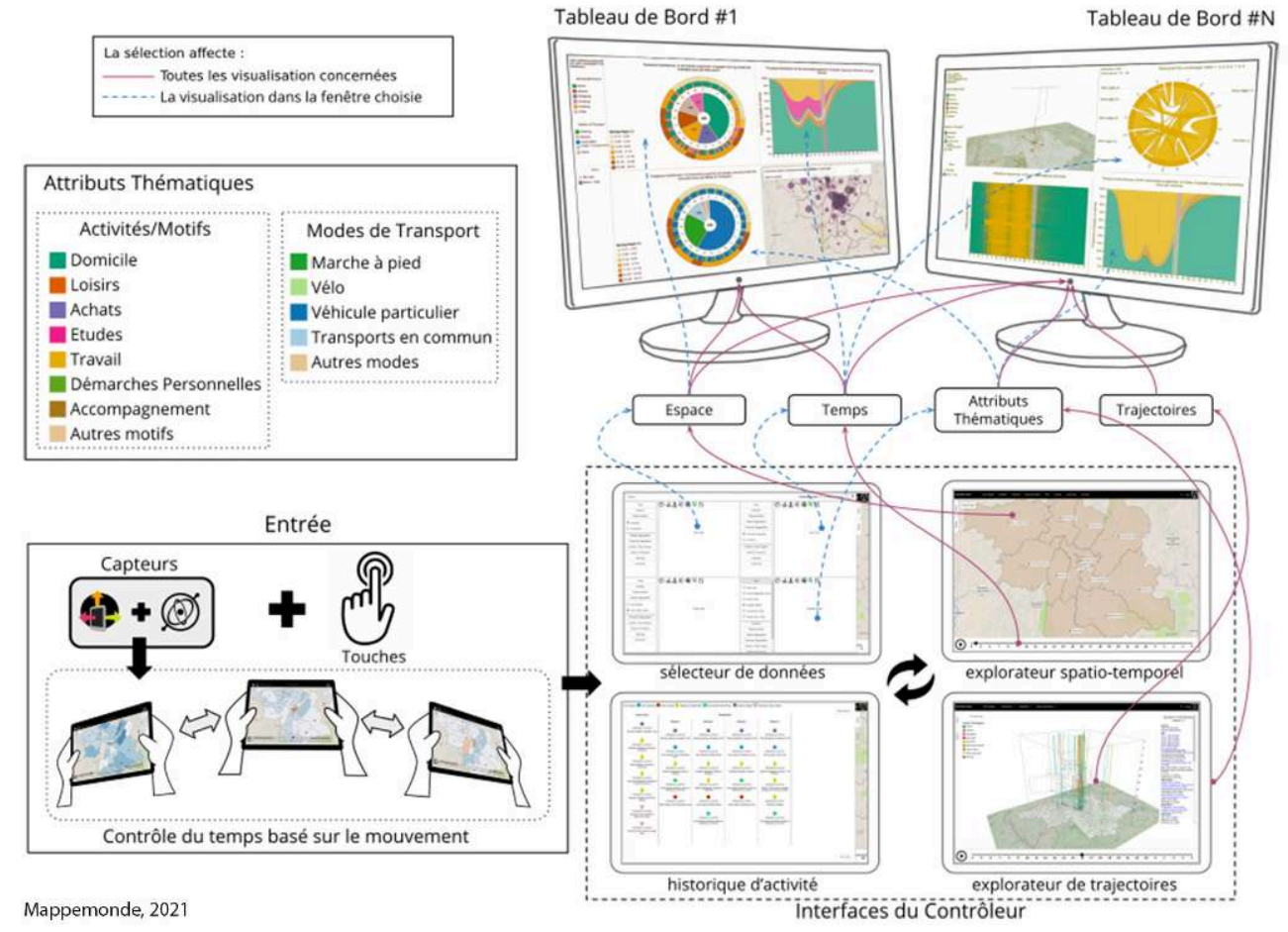

7 Les visualisations peuvent être exportées en format PNG et des informations descriptives sur chaque indicateur sont proposées pour chaque visualisation. L'environnement intègre un tutoriel qui permet à l'utilisateur d'appréhender les outils interactifs pour l'exploration des données.

Enfin, pour aider à l'exploration simultanée des dimensions spatiales et temporelles, nous proposons une technique d'animation tangible basée sur le mouvement de la tablette manipulée par l'utilisateur. Les angles d'inclinaison de la tablette correspondent aux périodes temporelles et l'animation cartographique associée est contrôlée via le mouvement effectué par l'utilisateur (Menin et al., 2018). 
Figure 8. Architecture fonctionnelle de l'environnement eSTIMe

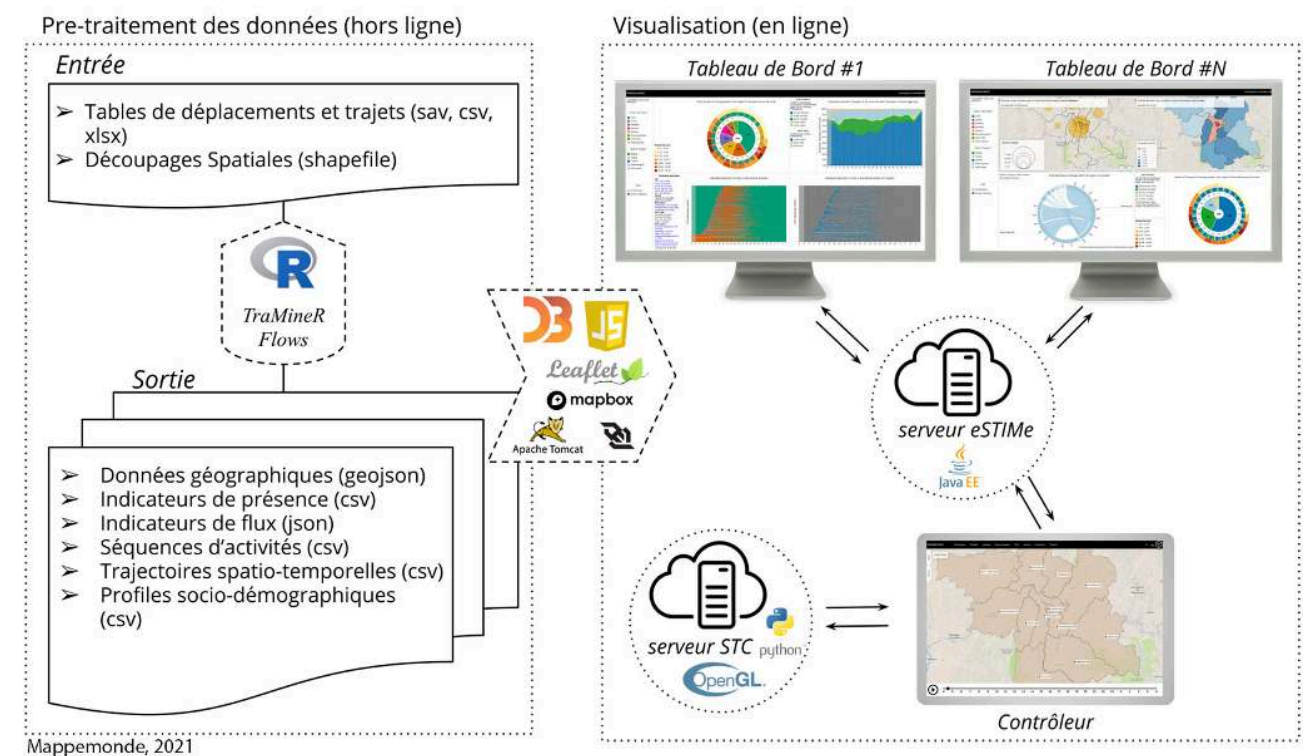

9 L'architecture d'eSTIMe (voir figure 8) est basée sur des technologies du Web. Le côté serveur s'appuie sur la technologie WebSocket et est structuré en deux modules: le premier développé en Java gère la communication entre les multiples écrans : le second développé en Python, utilise la bibliothèque OpenGL pour générer le graphique 3D du cube spatio-temporel. Le côté client est développé en JavaScript et utilise les bibliothèques D3 (Data-Driven Documents) et Leaflet pour générer, respectivement, les graphiques et les représentations cartographiques en 2D. Les données sont stockées localement sous la forme de fichiers geojson pour les informations spatiales, json pour les données de flux, et csv pour les autres. Les données sont prétraitées par des scripts $\mathrm{R}$ qui utilisent, parmi d'autres, les packages Flows et TraMineR. Ce dernier sert à créer la typologie de séquences.

\section{Bilan et Perspectives}

Tout au long du développement d'eSTIMe, des expériences ont été menées auprès des utilisateurs pour évaluer son ergonomie et son efficacité en termes d'analyse (Menin et al., 2019). Notre approche a été jugée positivement, car elle offre une diversité d'analyses visuelles des données de mobilité. eSTIMe est l'un des premiers outils de géovisualisation à offrir un environnement où sont intégrées des représentations permettant

-à la fois des analyses au niveau agrégé (cartes, roue de la mobilité, diagramme de flux, chronogramme) et au niveau individuel (actogramme, cube spatio-temporel) ;

- de naviguer d'un point de vue à l'autre (flux et déplacements, territoire et trajectoires quotidiennes) au sein d'un même environnement ;

- de faire varier la granularité spatiale à laquelle sont présentés les indicateurs et de les comparer aux différentes heures de la journée et en fonction de plusieurs attributs thématiques ;

- l'exploration des indicateurs sur le temps à travers trois techniques indépendantes: (1) l'animation, au moyen d'une interface basée sur le mouvement d'inclinaison d'une tablette (2) la juxtaposition, en affichant côte à côte des indicateurs pour différents créneaux 
horaires au moyen de la technique de figement du temps et (3) le cube spatio-temporel qui permet la visualisation des trajectoires quotidiennes dans le temps et l'espace simultanément.

eSTIMe peut être facilement utilisé avec d'autres jeux de données dans la mesure où leur format permet leur structuration en matrices d'information de flux, de présence et de séquences. Même si les attributs thématiques pour décrire les déplacements sont une information précieuse pour l'analyse des mobilités quotidiennes, eSTIMe n'empêcherait pas l'exploration d'un jeu de données sans attribut thématique (par exemple, des traces GPS séquencées) avec des visualisations d'effectifs globaux. Enfin, l'environnement peut gérer plusieurs affichages en offrant autant d'espace que nécessaire pour explorer les données en détail. En outre, le système s'adapte à différentes configurations technologiques, par exemple le déport du Contrôleur commande sur un écran conventionnel, en cas d'absence de tablette, en utilisant la souris et le clavier au lieu des options tactiles. Ces modifications suppriment la possibilité d'animer le temps par l'inclinaison du dispositif, mais la comparaison est toujours possible grâce à la juxtaposition des temps et à la sélection directe des unités de temps sur la ligne du temps.

\section{BIBLIOGRAPHIE}

ANDRÉ-POYAUD I., CHARDONNEL S., CHARLEUX L., TABAKA K. (2006). « La mobilité au cœur des emplois du temps des citadins ». In Y. CHALAS, F. PAULHIAC. La mobilité qui fait la ville, Lyon : CERTU, p. 67-95. BAHOKEN F. (2011). « Représentation graphique des matrices. Graphe et/ou carte des flux?». BERTIN J. (1983). Semiology of graphics; diagrams networks maps. University of Wisconsin Press. CEREMA. (2010). Enquête Ménages Déplacements, Grenoble/Grande région grenobloise (EMD, Grenoble/ Grande région grenobloise). Syndicat mixte des transports en commun de l'agglomération grenobloise (producteurs), ADISP (diffuseur).

CEREMA. (2015). Enquête Ménages Déplacements, Lyon/Aire métropolitaine lyonnaise (EMD, Lyon/Aire métropolitaine lyonnaise). Syndicat mixte des transports pour le Rhône et l'agglomération lyonnaise (producteurs), ADISP (diffuseur).

CEREMA (2017). Enquête mobilité (EMC2) 2018. Rennes/Ille-et-Vilaine.

CHARDONNEL S., CHARLEUX L., THIBAUlt P. (2010). « Analyser les routines dans les emplois du temps par la mesure des concordances d'actogrammes ». In A. BANOS et T. THÉVENIN. Mobilités urbaines et risques des transports : approches géographiques, Hermès Lavoisier, p. 23-50. En ligne : https:// halshs.archives-ouvertes.fr/halshs-00531662

LESNARD L. (2010). "Setting cost in optimal matching to uncover contemporaneous socio-temporal patterns". Sociological Methods and Research, vol. 38, n 3. https://doi.org/ $10.1177 / 0049124110362526$

MENIN A., CHARDONNEL S., DAVOINE P. A., NEDEL L. (2019). "ESTIMe: Towards an all-in-one geovisualization environment for daily mobility analysis". Proceedings - 32nd Conference on 
Graphics, Patterns and Images, SIBGRAPI 2019, p. 39-46. https://doi.org/10.1109/SIBGRAPI. 2019.00014

MENIN A., CHARDONNEL S., DAVOINE P. A., NEDEL L. (2018). "Exploring Shifting Densities through a Movement-based Cartographic Interface”. GIScience, 48, p. 1-6.

MENIN A. (2020). eSTIMe : a visualization framework for assisting a multi-perspective analysis of daily mobility data. Université Grenoble Alpes. https://hal.archives-ouvertes.fr/tel-03081054 MENIN A., CHARDONNEL S., DAVOINE P.-A., ORTEGA M., DUBLÉ E., NEDEL L. (2020). « eSTIMe : une approche visuelle, interactive et modulable pour l'analyse multi-points de vue des mobilités quotidiennes ». Geomatica, vol. 74, n 3, p. 1-22. https://doi.org/10.1139/geomat-2020-0006 MIRANDA F., DORAISWAMY H., LAGE M., ZHAO K., GONÇALVES B., WILSON L., HSIEH M., SILVA C. T. (2017). “Urban Pulse: Capturing the Rhythm of Cities". IEEE Transactions on Visualization and Computer Graphics, vol. 23, no 1, p. 791-800. https://doi.org/10.1109/TVCG.2016.2598585

PISTRE P., COMMENGES H., GUERRERO D., PROULHAC L. (2015). « Définitions opérationnelles du temps pour l'analyse des données longitudinales : illustration dans le champ des mobilités spatiales ». Nouvelles perspectives en sciences sociales, vol. 10, $\mathrm{n}^{\circ} 2$, p. 199-236. halshs-01163931 ROBETTE N. (2011). Explorer et décrire les parcours de vie : les typologies de trajectoires. Paris : CEPED.

\section{AUTEURS}

\section{ALINE MENIN}

Univ. Grenoble Alpes, CNRS, Grenoble INP, LIG

\section{PAULE-ANNICK DAVOINE}

Univ. Grenoble Alpes, CNRS, Grenoble INP, LIG. Univ. Grenoble Alpes, CNRS, Science Po Grenoble, PACTE

\section{SONIA CHARDONNEL}

Univ. Grenoble Alpes, CNRS, Science Po Grenoble, PACTE

\section{MICHAEL ORTEGA}

Univ. Grenoble Alpes, CNRS, Grenoble INP, LIG

\section{ÉTIENNE DUBLE}

Univ. Grenoble Alpes, CNRS, Grenoble INP, LIG

\section{LUCIANA NEDEL}

Federal University of Rio Grande do Sul, Institute of Informatics, Porto Alegre, Brazil 